\section{RE-CONCEPTUALIZACIÓN DEL CONSTRUCTO DE IMAGEN CORPORAL DESDE UNA PERSPECTIVA MULTIDISCIPLINAR}

\author{
Nuria Castro-Lemus \\ Universidad de Sevilla \\ ncastro@us.es
}

\section{RECONCEPTUALIZATION OF THE BODY IMAGE CONSTRUCT FROM A MULTIDISCIPLINARY PERSPECTIVE}

Cómo citar este artículo/Citation: Castro-Lemus, N. (2016). Reconceptualización del constructo de imagen corporal desde una perspectiva multidisciplinar. Arbor, 192 (781): a353. doi: http:// dx.doi.org/10.3989/arbor.2016.781n5010
Copyright: (c) 2016 CSIC. Este es un artículo de acceso abierto distribuido bajo los términos de la licencia Creative Commons Attribution (CC BY) España 3.0.

Recibido: 23 marzo 2015. Aceptado: 2 mayo 2016

RESUMEN: El presente artículo realiza de manera sistemática un repaso de las principales conceptualizaciones que existen del constructo de imagen corporal desde diferentes disciplinas como la filosófica, psicológica, sociológica e incluso la Educación Física. Dicho análisis argumenta la unificación terminológica de términos como cuerpo, corporeidad, corporalidad, identidad corporal, autoconcepto, capital corporal y esquema corporal. Y ofrece una re-conceptualización propia del constructo.

PALABRAS CLAVE: cuerpo; corporeidad; corporalidad; identidad corporal; autoconcepto; capital corporal y esquema corporal; Sociología; Psicología; Educación y Educación Física.
ABSTRACT: The present article carries out a systematic review of the main conceptualizations that currently exist of the body image construct from different disciplines, such as philosophy, psychology, sociology, and even Physical Education. This review argues the terminological unification of terms such as body, embodiment, corporeality, corporal identity, selfconcept, corporal asset, and body schema. It also offers a reconceptualization of the construct.

KEYWORDS: body; embodiment; corporeality; corporal identity; self-concept; corporal asset; body schema; Sociology; Psychology; Education and Physical Education. 


\section{INTRODUCCIÓN}

La imagen corporal ha sido un tema de interés desde épocas prehistóricas, pero podríamos identificar un resurgir científico cuando Hilde Bruch (1962) revela la relación que tiene la imagen corporal con los trastornos de la conducta alimentaria (TCA), concretamente la anorexia. Uno de los referentes más importantes en el estudio y conceptualización de la imagen corporal es Slade, quien en su artículo titulado What is Body Image? Y publicado en la revista Behaviour Research and Therapy en 1994, realiza una de las primeras revisiones bibliográficas sobre la conceptualización del constructo que se convierte en referente a seguir a partir de entonces.

La literatura demuestra que la investigación científica básica y aplicada sobre la imagen corporal ha crecido constantemente durante el último medio siglo, con un aumento impresionante en las últimas décadas. Pruzinsky y Cash (2002) realizaron una búsqueda por algunas de las principales bases de datos internaciones como PsycINFO y PubMed y demostraron este aumento progresivo entre las décadas de 1970 a 1990. Exactamente registraron la siguiente proporción en cuanto a citas relacionadas con la imagen corporal general y con la (in)satisfacción corporal: en la década de 1970: 1.970 y 1.785 citas. En la década de 1980:1.428 y 1.785. Y en la década de 1990: 2.477 y 2.766 .

El auge suscitado ha quedado patente en el aumento de revistas científicas que se dedican en algún momento al abordaje de este tipo de temáticas (Cash, 2004). Contreras Espinosa y González Romo (2010) realizan un estudio que incluye una revisión realizada a 136 artículos publicados en 67 revistas de ámbito internacional, y destacan la tendencia que existe en relacionar los TCA con los medios de comunicación. Igualmente señalan la tendencia a utilizar en la mayoría de las muestras a mujeres jóvenes, lo que provoca carencias sobre todo en las investigaciones donde la muestra sea exclusivamente masculina o también aunque en menor grado las que incluyen chicos y chicas indistintamente y en la misma proporción.

De todas las revistas existentes en el mercado científico, debemos destacar la creación en el 2004 de la revista científica dedicada a la imagen corporal llamada Body Image: An International Journal of Research, de la que es editor y director Thomas F. Cash, personaje dedicado a su estudio desde hace más de 30 años, reconocido investigador por la publicación de multitud de investigaciones y la creación de instrumentos para la evaluación de algunas de las dimensiones de la imagen corporal (Cash, 2004).

Siguiendo en esta línea, hemos realizado una búsqueda por la base de datos TESEO y hemos encontrado un total de 31 tesis que abordan la imagen corporal como temática principal en los últimos diez años. De ellas podemos destacar que la mayor parte están relacionadas directamente con algún tipo de trastorno de conducta alimentario, con la práctica de ejercicio físico, con los medios de comunicación y con el diseño y validación de algún tipo de instrumento evaluativo de la imagen corporal.

Otra búsqueda a nivel internacional por la ProQuest Dissertations and Theses muestra que de las tesis publicadas en Estados Unidos en el año 2010, 59 abordaban la imagen corporal en sus distintas dimensiones y además lo llevaban reflejado en su título. En la misma base de datos aparecen nueve tesis publicadas en el mes de enero de 2011. Como ejemplos podemos destacar que la mayor parte de ellas tratan la insatisfacción corporal como temática central y la relacionan con distintas particularidades como los medios de comunicación (Belden, 2010; Ferguson, 2011) y la salud, ya sea como indicador de riesgo (Petty, 2011) o relacionada a su vez con la apariencia, el estado físico o teorías feministas (Cooper-Molinero, 2011; Rima, 2011). También destacamos el número de tesis que se dedicaron a relacionar la imagen corporal con algún tipo de práctica deportiva, como por ejemplo la que la relaciona con las actividades extraescolares practicadas en Secundaria (Joshi, 2011).

Pero, ¿qué es la imagen corporal? ¿A qué nos referimos cuando hablamos de imagen corporal? ¿Qué dimensiones tiene?

\section{PRIMERAS APROXIMACIONES}

Uno de los referentes más importantes que se tienen sobre la imagen corporal proviene de la neuropsicología. Paul Schilder publica en 1935 una monografía sobre la imagen y la apariencia del cuerpo humano en la que considera la imagen corporal no sólo como una estructura fisiológica sino también psicológica. Además considera la imagen corporal como un factor decisivo en toda la acción humana y como una parte constitutiva de la persona (Schilder, 1989). Este autor entendía que esta imagen del cuerpo no sólo se constituía por las impresiones recibidas a través de los sentidos, de los músculos (aponeurosis) o vísceras, sino que el cuerpo se percibía como una unidad más allá de las percepciones. En palabras 
de Schilder (1989) podemos decir que "el esquema corporal es la imagen tridimensional que todo el mundo tiene de sí mismo" y que podemos llamar a esta imagen "imagen corporal".

Ya entonces empiezan a confundirse los términos "imagen corporal" y "esquema corporal", llegando esta confusión hasta nuestros días. Estudios como el de Baile Ayensa (2003) aseguran que todavía no han sido consensuados por la comunidad científica muchos términos utilizados en diferentes ámbitos que son cercanos conceptualmente, similares en algunos aspectos o incluso sinónimos a "imagen corporal". Pero esta confusión conceptual no se centra exclusivamente en utilizar "esquema corporal" e "imagen corporal" indistintamente, sino que se aglutinan términos como cuerpo, corporeidad, identidad corporal, autoconcepto físico e incluso capital corporal. Y esta diversidad de terminología depende en muchas ocasiones de la disciplina desde la que abordemos el constructo de imagen corporal.

Según Grasso (2006), el abordaje de la imagen corporal implica un posicionamiento filosófico y a veces hasta teológico. Desde un punto de vista filosófico, se identifican dos enfoques de abordaje: el que considera el cuerpo como máquina-materia (dualismo) y el que entiende el cuerpo como corpóreo.

El dualismo ha tenido diferentes planteamientos a lo largo de la historia y de las diferentes culturas. Cruz y Maganto (2002) analizan esta evolución y describe un primer planteamiento que se daba en los pueblos antiguos. En las antiguas civilizaciones y en determinados posicionamientos teológicos, el cuerpo pertenecía a lo terrenal y el alma pertenecía a lo espiritual, a los dioses y poderes de la naturaleza. Así entiende que el verdadero sentido de la vida pasaba irremediablemente por conseguir proporcionar felicidad al alma (a los Dioses, a la Naturaleza). Este pensamiento antiguo evoluciona y la importancia de lo espiritual pasa a ser sustituido por lo mental. Este pensamiento, liderado por Descartes, entiende el cuerpo como materia-máquina separada de la mente pero supeditada a ella. Esto sugiere que cualquier modificación externa al cuerpo no sólo es posible sino que además tendrá repercusión "casi matemática" en la mente de la persona. Esta dualidad cartesiana tiene su legado, el cual llega hasta nuestros días.

Este abordaje dualista del cuerpo se ha visto reflejado a lo largo de la historia de la sociedad occidental. Los primeros indicios que tenemos es el utilizado tan- to por los antiguos egipcios (s. VII a.C.) como por los griegos (s. V a.C.). Ya en estos casos el objetivo era encontrar la proporcionalidad estética, el llamado canon de belleza. Los egipcios tomaban como referencia las proporciones corporales del faraón. Los griegos, en cambio, utilizaron las aportaciones de Platón y la de escultores como Policleto y Lísipo. Así establecieron que las dimensiones corporales estaban en proporción a las dimensiones de la cabeza (Martín Serrano, 2002; Galiano, Porta y Tejedo, 2003; Heredia y Espejo, 2009; García Alonso, 2011).

Más tarde, en el Medievo, el valor del cuerpo queda relegado a un último plano, por lo que existe un parón científico en este sentido. No es hasta el Renacimiento que, aunque se sigue teniendo la misma concepción dualista del cuerpo que en el Medievo, el cuerpo pasa a ser considerado como una máquina. Una máquina perfecta hecha a semejanza de Dios, considerado como la pura esencia del concepto de belleza natural. En esos momentos se realizan multitud de estudios anatómicos que sirven para dar comienzo a lo que sería el modelado del cuerpo (Heredia y Espejo, 2009; García Alonso, 2011).

Desde un punto de vista fenomenológico, materia y cuerpo no pueden ser el mismo término, ya que, aunque hagan referencia a las mismas realidades, denotan distintos significados. El cuerpo, al igual que la materia, tiene como propiedad el hecho de ocupar lugar en el espacio. Pero no podemos describir ningún ser humano sólo con la palabra "cuerpo", ya que estas propiedades que existen en el cuerpo no son el cuerpo en sí. La palabra cuerpo puede ser definición individual o esencia particular de cualquier cosa en el entorno real. Esto es así porque su identidad de unicidad irrepetible implica consideraciones o notas propias que distinguen a un individuo de los demás. Por lo tanto, el término "cuerpo" resulta impreciso e inexacto para cualquier entidad o cosa (Astacio, 2001).

La dificultad del cuerpo atañe a la naturaleza compleja del mismo. Éste tiene multitud de potencialidades y posibilidades inciertas desde el que se lleva a cabo la materialización o desde la encarnación de los contenidos de fondo que animan los ejes culturales de cada momento. En palabras de Sierra Hernando (2007), ni el cuerpo es una realidad unitaria y completa, ni estática, ni substancial. Podríamos decir que el cuerpo no resulta el depositario natural de la acción humana sino el principio que la dimensiona, que le da mayor dimensión o importancia a la acción humana, al movimiento. 


\section{LA IMAGEN CORPORAL DESDE UNA PERSPECTIVA MULTIDISCIPLINAR}

El abordaje de la imagen corporal desde distintas disciplinas ha enriquecido y matizado este constructo. Desde el campo de la Literatura hasta la Filosofía, Antropología, Psicología, Psicoanálisis, Sociología, Educación Física y Deporte, etc. (De la Cruz Vives, 2002; Pastor Pradillo, 2002; Grasso, 2006; Ruiz Calvente, 2008).

\section{Desde la sociología}

Merleau-Ponty, uno de los más importantes representantes del enfoque fenomenológico, aborda la imagen corporal desde la sociología y considera que es nuestro cuerpo lo que asegura que existan objetos para nosotros. Por lo tanto, el cuerpo no es un objeto cualquiera del mundo, es un medio de comunicación entre nosotros y el mundo. Además, concibe dos dimensiones del cuerpo: como estructura experiencial vivida (Yo no estoy delante de mi cuerpo, estoy en mi cuerpo, o mejor, soy mi cuerpo) y como ámbito de los mecanismos cognitivos (percibir es tornar presente cualquier cosa con la ayuda del cuerpo) (Firenze, 2003; Costa, 2006; Pérez Riobello, 2008).

Apoyándonos en la perspectiva fenomenológica de Merleau-Ponty se puede entender la corporeidad como una dimensión global y compleja de la persona. Sería la construcción permanente de la persona en su esfera biológica, psicológica y sociocultural. Abarca la unidad de lo psicofísico, lo espiritual, lo motriz, lo afectivo, lo social y lo intelectual, a partir de lo que tiene significado para él y para la sociedad. Dos nuevos términos aparecen relacionados, en este caso con la corporeidad: "habitus", término sociológico que se utiliza como sinónimo en muchas ocasiones de imagen corporal, y capital corporal (Sierra Hernando, 2007; Barbero González, 2007).

Según Pierre Bourdieu, el "habitus" es la historia incorporada y a la vez puesta a disposición en cada situación para percibir, ser, evaluar, etc. Este "habitus" integra todas las expectativas pasadas y funciona en cada momento como matriz estructurante de las percepciones, las apreciaciones y las acciones de las personas cara a una coyuntura o acontecimiento y que contribuye a la reproducción de modelos. El cuerpo es historia y el "habitus" es la historia hecha cuerpo (Grasso, 2001; Barbero González, 2007; Rodríguez y Galak, 2009).

A nivel sociológico podemos afirmar que todas las actividades humanas son corpóreas, no se dan sin el cuerpo, ni las relaciones humanas intrapersonales, de comunicación, copulación o socialización; ni las relaciones con el medio natural sensible y manipulable, ni las relaciones intrapersonales de reflexión y opción. El cuerpo se constituye como centro de gravedad donde se produce la gran síntesis de todas las fuerzas interpretativas de lo real en una cultura dada. Se ve el cuerpo como la auténtica cultura social (Sierra Hernando, 2007).

Por otro lado, el cuerpo como elemento de interacción social ha ido cobrando un estatus capital en nuestras sociedades; el cuerpo como plusvalía social y como elemento de movilidad social. Bourdieu entiende este capital como todo aquello que puede producir efecto en la competencia social, cualquier instrumento de apropiación de oportunidades que permite la dominación. El capital corporal, en cuanto forma de capital cultural (socialmente construido e íntimamente ligado a las condiciones materiales y culturales de existencia colectiva e individual, etc.), nos permite cuestionar el carácter universal y asocial con el que con frecuencia explicamos los valores y la importancia del cuerpo y del movimiento (Barbero González, 2005).

Un ejemplo de capital corporal lo encontramos en el análisis que realiza Carrión (2010, 8 de febrero) de la nueva versión de Sherlock Holmes. La escena clave de la película es el combate clandestino que protagoniza Sherlock Holmes. Su lucha no es por honor ni por dinero. En la lógica psicológica del nuevo personaje, el combate se debe a una mezcla de terapia y de impulso autodestructivo. En la lógica narrativa de la película, quitarle la camisa al detective, convertirlo en un luchador implacable, significa subrayar la importancia de su cuerpo en esta nueva etapa de su secular singladura. Aunque la trama dependa de la capacidad deductiva de Holmes, la película avanza en clave de película de acción. El cuerpo es el trampolín desde el que salta la mirada para atar cabos. La inteligencia se somete al vértigo corporal. No podría ser de otro modo en una reencarnación del siglo XXI".

El capital corporal sería todo lo que es el cuerpo, posee el cuerpo y ha ido adquiriendo con el tiempo: la experiencia, la cultura, el entorno que le rodea, etc. Hablamos de constructo multidimensional en el que intervienen diferentes factores sociales, históricos, culturales, etc., al igual que cuando hablábamos de cuerpo desde una perspectiva fenomenológica (Córdoba, 2010; Aréchaga, 2010). 


\section{Desde la psicología}

La psicología ha sido y es otra de las disciplinas que abordan la imagen corporal y su relación con el autoconcepto. Uno de los referentes más importantes lo tenemos en el grupo de trabajo dirigido por Goñi del Departamento de Psicología Educativa de la Universidad del País Vasco. Este grupo lleva trabajando sobre el autoconcepto desde los años noventa desde diferentes perspectivas. De ahí la producción de tesis doctorales como por ejemplo: "Análisis correlacional y eficacia en la mejora del autoconcepto físico de un programa de intervención" (Zulaika, 1999); "Elaboración y validación del cuestionario AFI (Autokontzeptu Fisikoaren Itaunketa) de autoconcepto físico" (Esnaola, 2005); "El autoconcepto físico: estructura interna, medida y variabilidad" (Ruiz de Azúa, 2007); "El autoconcepto físico y el bienestar/malestar psicológico en la adolescencia" (Rodríguez Fernández, 2008); "Actividad física y autoconcepto físico en la edad adulta" (Infante, 2009); "El autoconcepto personal: estructura y variabilidad" (Goñi, 2009); "El autoconcepto social: componentes, desarrollo y medida" (Fernández Zabala, 2010); "La presión sociocultural percibida sobre el autoconcepto físico. Medida y variabilidad" (González Fernández, 2011); “Diseño y evaluación de un programa educativo para la mejora del autoconcepto físico" (Axpe, 2012); "El autoconcepto físico y la motivación físico-deportiva durante la adolescencia" (Revuelta, 2012); "Características físicas y psicológicas de jóvenes futbolistas de alto rendimiento" (Arostegi, 2013) y "El autoconcepto musical, motivación y bienestar psicológico del alumnado de Conservatorio" (Zubeldia, 2015). Estas investigaciones, a su vez, han producido artículos científicos divulgados en revistas de impacto (Goñi Grandmontagne, Ruiz de Azúa García y Rodríguez Fernández, 2005; Esnaola, 2008).

La relación puede ser explicada a grandes rasgos con la conceptualización del autoconcepto. Éste se compone de un componente social, otro académico y otro de la persona o físico. Este último componente es el que estaría directamente relacionado con la imagen corporal y con la autoestima (Esnaola y Revuelta, 2009).

En un estudio realizado por Rodríguez Fernández (2009) se asegura incluso que el autoconcepto físico guarda una relación de signo positivo con el bienestar psicológico y una relación negativa con el malestar psicológico. En este sentido, cuanto más elevado sea el yo-físico percibido, mayor bienestar experimentará la persona, menores niveles de ansiedad y depresión o menor riesgo tendrá de padecer un trastorno de la conducta alimentaria (Rodríguez Fernández, 2009).
En términos generales podríamos decir que el autoconcepto se define como la totalidad de las percepciones que cada persona tiene de sí misma. Esnaola (2008) realiza una revisión bibliográfica y define el autoconcepto en base a siete aspectos. Se trata de una estructura dinámica, multidimensional, jerárquica, estable, que aumenta su dimensionalidad con la edad y como percepción de uno mismo.

Presenta tanto aspectos descriptivos como evaluativos. Éstos serían juicios de valor a cerca de la percepción. Los descriptivos harían referencia a cómo somos en cuanto a edad, sexo, profesión y/o rol social, características físicas, modos de comportamiento, lazos sociales, etc. $Y$ los juicios evaluativos se refieren al aprecio o valoración que nos merece cada una de nuestras características así descritas. En estos juicios la persona expresa cómo se representa, conoce y valora a ella misma. Aunque a menudo se usan de manera equivalente autoconcepto y autoestima, se tiene establecido por la comunidad científica que la autoestima sería el aspecto evaluativo del autoconcepto físico (Cardenal y Fierro, 2003).

Se entiende el autoconcepto como la percepción que el individuo tiene de sí mismo y, en términos específicos, las actitudes, sentimientos y conocimiento de sí mismo acerca de sus habilidades, destrezas, apariencia física y la aceptación social (Faria, 2005).

Según Raich (1994), el principio del autoconcepto implica percepción y está compuesto por diferentes áreas; la social, la académica/laboral, la familiar, la corporal y la global. En palabras de Esteve Rodrigo, Musitu Ochoa y Lila Murillo (2005), el autoconcepto físico es una representación mental que se elabora al integrar la experiencia corporal y los sentimientos y emociones que ésta produce. Esta representación mental es multidimensional y jerárquica y está conformada por dos grandes subdominios o áreas que son: la apariencia física y la habilidad física.

\section{Desde la educación}

A nivel educativo, la imagen corporal adquiere vital importancia porque forma parte de la identidad de los educandos. De manera general podemos decir que a raíz de la postmodernidad, la identidad adquiere un gran protagonismo como constructo pedagógico. Esta identidad se desarrolla gracias a la construcción de un yo personal y social a través de procesos de reconocimiento e identificación de valores. La identidad implica, por tanto, la asunción de determinados valores históricos, culturas, ideas, etc. La convergencia 
de elecciones diferentes en éstas u otras dimensiones lleva a la idea de una identidad multidimensional, producto de la combinación e integración de todas ellas. Ello origina y da lugar a múltiples identidades: identidad personal, identidad cultural, identidad lingüística, religiosa, de género y la identidad corporal (Colás Bravo, 2007).

Esta identidad corporal y social forma parte del ser humano desde épocas prehistóricas. La literatura indica que en esos momentos los ideales de belleza estarían más influidos por causas funcionales primarias-básicas que por un hipotético sentido del cuerpo humano. La mayoría de las representaciones, dibujos, grabados, estatuillas, etc., de la figura humana de esa época debieran interpretarse generalmente como muestras alegóricas, de unas características morfológicas y sexuales que tendían a resaltar aspectos o funciones vitales de la vida cotidiana. Un claro ejemplo de canon de belleza funcional sería la Venus de Willendorf, cuya belleza residía y estaba resaltada por su potencial capacidad de fecundación y alimentación del recién nacido. En las antiguas civilizaciones, estos ideales de belleza se llamarían cánones de proporcionalidad y según la cultura tendría un significado $u$ otro. Los cánones hindúes y europeos marcarían estado de salud y en el antiguo Egipto o Grecia estatus social y cultural (García Alonso, 2011).

Más adelante, la espiritualidad copó los intereses de los transmisores de cultura $y$, a través de la religión, concretamente del cristianismo, se difundieron ideales de belleza que pretendían el sometimiento del cuerpo sobre el alma. Así aparecen los primeros casos de anorexia, identificados como "Anorexia Santa" (Heredia y Espejo, 2009).

Pero, el verdadero impacto que supone la imagen corporal en la sociedad actual comienza con la revolución tecnológica. Podemos decir que con anterioridad, la transmisión de los ideales corporales se producía a través de la escritura, la escultura o pintura y estos ideales llegaban allí dónde llegaban estas obras. Uno de los hitos más importantes en cuanto a modelos corporales a imitar, se produjo con el invento de la fotografía y con el desarrollo de la industria cinematográfica. Con esta revolución tecnológica nace el star-system y los pin-up, modelos de estrella cinematográfica que se creaban como reclamo publicitario de películas. Estos y estas modelos llegaban a ser considerados ídolos, iconos y objeto de deseo a imitar por el resto de la población. El momento histórico y la localización donde aparecen estos modelos a imitar son Estados Unidos, tras la I Guerra Mundial. Esta época resulta un momen- to de esplendor, por lo que estos ideales reflejaban que lo grande era bello. Por lo tanto, podríamos decir que el ideal que favorecían no supuso graves alteraciones a la hora de imitarlo. Sin embargo, en la actualidad, debido a la revolución tecnológica y a los cánones de belleza establecidos, estos modelos de referencia están cambiando peligrosamente los pensamientos y actitudes de gran parte de la población. Sobre todo, los de los y las adolescentes (Martín Serrano, 2002; Galiano, Porta y Tejado, 2003).

Como podemos observar, la identidad corporal se construye de forma dinámica y evolutiva. Es un proceso dinámico con posibles grados de evolución en el tiempo. Desde un punto de vista psicológico tenemos que tener en cuenta un tiempo objetivo y un tiempo subjetivo, y en la construcción de la identidad deben conjugarse estos dos tiempos. Estos ejes temporales marcan trayectorias de identidad diferenciadas en los sujetos. El tiempo pasado, presente y futuro actúa como eje articulador en los procesos identitarios. Las experiencias vitales previas, las condiciones existenciales presentes y las expectativas futuras son referentes importantes para comprender las transformaciones y cambios de identidad. La identidad no es algo unitario, fijo y estable, sino que está en constante construcción y cambia según las circunstancias (Colás Bravo, 2007).

Encontramos una necesidad de trabajar esta identidad corporal desde el ámbito educativo y, en especial, desde la Educación Física.

\section{Desde la Educación Física}

El cuerpo como eje vertebrador de la Educación Física ha sido ampliamente estudiado. Vázquez (2001) se plantea las aportaciones recientes de las ciencias sociales y humanas referentes al cuerpo y opina que, aunque más imprecisas, son más próximas a los intereses actuales. Ella concibe el cuerpo como una entidad psicosomática que sobrepasa el mero concepto biologicista y rompe el tradicional dualismo mente-cuerpo. Ella entiende que el mismo cuerpo tiene diferentes facetas como cuerpo acrobático, el cuerpo pensante y el cuerpo comunicación. Además, realiza un símil con el tipo de educación que conllevaría, siendo educación físico-deportiva, psicomotriz y la expresión corporal, respectivamente (Vázquez Gómez, 1989).

Pastor Pradillo (2002) defiende que las líneas más importantes que en la actualidad sirven de orientación a la mayoría de las propuestas que pretenden una definición conceptual del cuerpo, son: 
- El cuerpo como generador de actividad.

- El cuerpo adaptativo y funcional.

- El cuerpo que se deduce de la propia experiencia vital.

Al intentar conceptualizar, aclarar y distinguir el concepto "cuerpo" de otros conceptos o constructos afines, hablamos de un constructo multidimensional en el que influyen aspectos biológicos, sociales, culturales, políticos y afectivos. Las nuevas concepciones del cuerpo, como elemento fundamental de la personalidad y de la relación con los demás, así como las teorías unitarias con el ser humano, rebasan el concepto cuerpo-máquina y dan lugar a otros paradigmas. Destacan del cuerpo su capacidad como medio o instrumento de expresión de lo humano. Con esta intención, algunos autores proponen lo "corpóreo" como contenido principal de esta nueva comprensión del cuerpo (Grasso, 2006; Benjumea Pérez, 2010).

Según Pastor Pradillo (2008), los paradigmas que a lo largo de la historia se han utilizado para determinar el significado o significados del constructo cuerpo se han concretado en diversos términos que, de forma paralela, han relacionado su común evolución conceptual. El autor entiende que ha habido una sustitución del paradigma que describe la naturaleza humana como un modelo dualista por otro unitario y lo califica de "corte epistemológico". Este cambio obliga a ensayar nuevas consideraciones y a incluir contenidos distintos y más diversificados. Para expresarlo se ha recurrido, por ejemplo, a términos como carne, cuerpo, lo corporal, corporalidad o corporeidad, para finalizar en la actualidad con la asunción de la noción de "Yo corporal".

Otros autores opinan que el trabajo motriz se sustenta en la concepción de la existencia de un sistema estructural del cuerpo que se compone de un cuerpo biológico, social, cultural, político y afectivo en continua transformación dentro del continuum espaciotiempo. Esta argumentación defiende el trabajo de la motricidad en base a que no es lo mismo tratar con un cuerpo que pertenece a una persona y quiere conseguir algo de él, que trabajar con un cuerpo que es una persona. No es lo mismo sentir a través de un cuerpo, que sentir con el cuerpo (Dewar, 1994; Pazos y Rey, 2000; Grasso, 2001; Eisenberg, 2003).

Apoyado en esta visión de lo corporal, Le Boulch (2001) entiende que para el desarrollo integral de la persona debemos tener en cuenta que el cuerpo propio es a la vez cuerpo afectivo y cuerpo operativo. Argumenta en su teoría que cuando el aprendizaje motor adopta la forma de una mecanización, convierte nuestro propio cuerpo en un ente extraño para nosotros mismos. Así Le Boulch se posiciona ante la visión fenomenológica, evita la alternativa dualista y se niega a elegir entre el cuerpo objeto o el cuerpo sujeto. Entiende que el cuerpo, en función de su entorno, expresará sus emociones o será un instrumento eficaz de adaptación. Asume que la corporeidad es un elemento de la motricidad porque contribuye a comprender la experiencia del cuerpo propio, cuerpo vivido, experiencia que se da ante todo como un sentir, fusión o indistinción entre cuerpo y yo, y no como un simple proceso paralelo. Esta concepción es la que asume la motricidad. La motricidad hace suya la concepción de este elemento constitutivo y permite entender que más que hablar de cuerpo, es más pertinente ser cuerpo, lo cual, a su vez es hablar de motricidad (Benjumea Pérez, 2010).

El cuerpo se ha mostrado tradicionalmente como un misterio central donde se vislumbran las bases expresivas de toda cultura. Lo corpóreo fuerza a la existencia de "estar en el mundo" a materializar la experiencia humana y el pensamiento colectivo. La corporeidad implica la inserción de un cuerpo humano en un mundo significativo, en la relación dialéctica del cuerpo consigo mismo, con otros cuerpos y con los objetos (Pazos y Rey, 2000; Sierra Hernando, 2007; Benjumea Pérez, 2010).

Quizás para el idioma alemán resulta más sencilla esta diferenciación, ya que poseen dos palabras diferentes para referirse a cuerpo: Köper y Leib. El primero hace referencia al cuerpo como realidad material, que se constata mediante los sentidos. Leib sería el cuerpo como objeto de una experiencia compleja y unitaria. Leib es "la condición corpórea", más que el cuerpo considerado como realidad del orden natural. La traducción más exacta de Leib sería "corporeidad" (Rigobello, 2000).

En esta misma línea, Vázquez (2001) pone de manifiesto su versión: el cuerpo objetivo (conjunto de órganos, funciones, etc., objeto de las ciencias médicas) y el cuerpo subjetivo (cuerpo propio que sólo se alcanza por la introspección y la experiencia propia). Destaca también las aportaciones de diferentes autores que siguen en esta línea (Vázquez Gómez, 1989):

- Extracuerpo/Intracuerpo de Ortega y Gasset.

- Cuerpo/Corporeidad en López Ibor.

- Cuerpo para otro/cuerpo para mí en Sartre.

- Cuerpo objetivo/Cuerpo vivido en Merleau-Ponty. 
En este sentido, se diferencia la realidad física del cuerpo y se pueden destacar las nociones psicológicas tales como la conciencia, lo volitivo, lo cognitivo, lo afectivo o lo expresivo. Además no sólo se "posee" un cuerpo (que sólo hace), sino que su existencia humana implica hacer, saber, pensar, sentir, comunicar y querer (Trigo, 2000).

Si cuando hablamos de corporeidad expresamos una idea, un concepto múltiple, plural, complejo y diverso de una unidad, de uno mismo, ese uno que a su vez no tiene otro igual, que como individuo es único e irrepetible, y que como grupo, es un conjunto de sujetos absolutamente diversos; entonces, cuerpo y corporalidad o corporeidad no son lo mismo. Cuerpo debería ser entendido como una realidad objetiva que, a la manera de un objeto, posee una estructura delimitada, y corporeidad como una historicidad determinada. Esto último permite sostener que la corporeidad obedece a una construcción cultural, social e histórica. Además que el cuerpo vive o se expresa por medio de su corporeidad (Grasso, 2008; Sossa, 2009).

Benjumea Pérez (2010) realiza un estudio sobre la motricidad humana y entiende que ésta no puede existir sin corporeidad. Ve la motricidad como elemento inherente a la corporeidad. Ella sostiene que lo humano conlleva substancialmente un cuerpo. La corporeidad permite ver que lo humano no se limita al cuerpo fisio-biológico, aunque sí lo incluya significativamente. Sostiene que para entender la relación que existe entre motricidad y corporeidad se debe entender ésta como la consciencia que se tiene de sí como sujeto, a la vez que se configura en una unidad significante del ser humano que da sentido y que permite integrar todas las dimensiones del ser. Pastor (2002) entiende que, dentro de la "unidad ontológica de la persona", la corporeidad se manifestaría como un componente esencial respecto a la subjetividad humana, la cual debería entenderse como un resultado psicofísico.

Grasso (2001, p. 19) entiende que la corporeidad "es una concepción que rompe el habitus corporal que tiene incorporado el profesorado, que busca la identidad corporal del alumnado".

Pero este cuerpo "historizado" es el que Vázquez (2001) entiende que está singularizado, no sólo por este "habitus", sino también por el contexto en el que se desenvuelve, localizado en un lugar y momento histórico determinados. Se puede decir que el cuerpo es una construcción social, ya que las funciones corporales que sostienen la vida social de una persona muestran adquisiciones que no son innatas, sino que dependen del desarrollo según direcciones culturales precisas (Barbero González, 2007).

En esta línea, Barbero González (2007), apoyado en las teorías de Bourdieu, se plantea desvincularse del paradigma mecanicista en el que se glorifica al cuerpo como máquina y entiende que éste debe ser tratado de una forma más holística. Para su abordaje concibe al cuerpo como un "capital" que son los recursos, los dominios y el poder que tienen las personas, que les ayuda a "abrir puertas" e incrementar sus posibilidades vitales. El cuerpo es objeto (en la sociedad occidental actual) de múltiples inversiones (cosmética, dietética, vestimenta, quirúrgica, gimnástica, etc.) mediante las cuales intentamos mantener al cuerpo (re) presentable adecuándolo a los patrones hegemónicos del momento. Entenderemos, según este autor, capital corporal como una forma de capital cultural cuya posesión permite ejercer un poder real o simbólico.

En la construcción de identidades, el cuerpo se presenta como un foco, "locus" u objeto imprescindible de intervención. Cualquier tipo de iniciativa o discurso activados por cualquier agente, grupo o instancia social con una mínima capacidad de influencia, repercute inevitablemente sobre la vertiente corporal (Barbero González, 1998).

Algunas autoras dicen que la imagen corporal es el fundamento de la identidad personal, entienden que nada es más personalmente real que el propio cuerpo. La identidad supondría, por una parte, "identificar", es decir, "singularizar" algo de los demás y, por otra, "pertenecer", ubicación de los sujetos en un espacio común compartido (Dewar 1994; Grasso, 2001; Marín, 2002).

Grasso (2001) entiende que la identidad corporal es conocer y entender la corporeidad, distinguir y comprender lo que se constituye como figura visible, la apariencia física, la esencia intelectual y emocional, la representación de nuestro propio cuerpo. La identidad corporal nos ayuda a formarnos una idea de nuestro cuerpo a través de asociaciones, memoria, experiencias, intenciones y tendencias (Kogan, 2011).

Pero sin duda alguna, si hay dos términos que tienden a la confusión son la imagen corporal y el esquema corporal. Y es que, aunque autores como Dolto (1994) afirmaron rotundamente que el esquema corporal no era la imagen corporal. Lo cierto es que este término resulta ambiguo y difícil de precisar, por lo que muchas veces tiende a la confusión. Su estudio desde diferentes disciplinas ha ayudado 
y/o enturbiado la concreción del término (Le Boulch, 2001; Baile Ayensa, 2003; Sassano, 2003; Gallego del Castillo, 2009).

Existen incluso otros autores que añaden a esta confusión terminológica una tercera, como es la conciencia corporal. Ellos diferencian la imagen corporal del esquema corporal en que el primero aborda el nivel cualitativo (concepción subjetiva del propio cuerpo, condicionada por las características físicas de la persona y que se desarrolla de manera paralela a la concepción objetiva que los demás establecen de nuestro cuerpo). El esquema corporal abarcaría el nivel cuantitativo y lo definen como el tipo de adaptación que se establece entre la estructura ósea y los grados de tensión muscular del organismo, haciendo posible la percepción global y segmentaria del propio cuerpo. Estos autores mantienen que la conciencia corporal sería la noción fáctica del cuerpo como resultado de la existencia continuada de los dos conceptos anteriores. El esquema corporal es la experiencia propioceptiva, cenestésica, sinestesia, cenestésica, etc., que cada ser construye sobre la base del cuerpo propio (López Sáenz, 2002).

Le Boulch (2001) afirma rotundamente que esquema corporal e imagen corporal son los mismos términos y que los utiliza según se refiera a un análisis más neurológico o psicológico. Entiende que la imagen corporal es la emergencia mental o funcional del esquema corporal. Tanto el esquema como la imagen corporal representan para él una estructura integradora central de la personalidad. Sin embargo, cuando intenta definir o aclarar la terminología realiza una diferenciación clara. Por un lado, entiende que el esquema corporal es un concepto neurológico que corresponde al conjunto de las estructuras nerviosas que tratan la información propioceptiva. Añade que al depender esta estructura tanto de informaciones exteroceptivas, relativas al cuerpo-vivido, como a la función simbólica verbal, considera el esquema corporal como "una intuición de conjunto o un conocimiento inmediato que tenemos de nuestro cuerpo en relación de sus diferentes partes entre ellas y sus relaciones con el espacio circundante de objetos y personas". Por otro, cuando hace referencia a la imagen corporal la describe como por encima del esquema corporal, concretamente como un paso más hacia el cuerpo operativo (López Sáenz, 2002).

Gallego del Castillo (2009) realiza un estudio respecto a la evolución del término por entender que existe un problema taxonómico en su definición. Estructura su investigación en las aportaciones que se han reali- zado desde dos grandes perspectivas: una la fisioneurología y otra la psicología.

Respecto a la perspectiva fisioneurológica, destaca las diferentes aportaciones de autores que van desde Bonnier, Pick, Head, Schilder o Ajuriaguerra, entre otros. Podemos destacar al respecto que sus aportaciones no sólo van encaminadas a definir o describir el esquema corporal como la imagen de uno mismo o la imagen del cuerpo, sino que también explican la inseparable relación con los estímulos vestibulares, mecanismos cerebrales, experiencias sensoriales, espaciales, etc.

Respecto a la perspectiva psicológica, psicoterapéutica y psicosomática, destaca que están bastante relacionadas con los estudios de enfermedades mentales que afectan al cuerpo y al movimiento, principalmente. Resalta las aportaciones de la psicología genética de Piaget, Walon y Pierón, que hablan de la importancia de la relación entre el individuo y el medio para la formación del esquema corporal. En la psicología de la percepción subraya la importancia que le dan a la percepción de los objetos circundantes para la percepción del cuerpo.

Grasso (2005) identifica los dos términos como representaciones mentales del cuerpo. Sin embargo concreta que el esquema corporal sería "el cuerpo actual, en el espacio, la experiencia inmediata, a un cuerpo identificado en su forma, en el contenido de sus superficies limítrofes, situado, localizado como objeto percibido, orientado en un espacio". La imagen corporal sería un añadido de estas percepciones que influirían en las actuaciones. Pone el ejemplo de cuando nos encontramos un charco de agua en la calle. Antes de saltarlo, sabemos si podemos o no saltarlo por el conocimiento que tenemos de nuestro esquema corporal, por la organización sensorial: el largo de las piernas, la fuerza, la longitud del charco con respecto a su tamaño, etc. Podemos saber en este caso que somos capaces de saltarlo, sin embargo puede que no lo hagamos por alguna imagen negativa que tengamos de nosotros mismos. Podemos creer que nuestro cuerpo no es lo suficientemente hábil, que se puede resbalar o caer, debido a la influencia recibida por otras personas, a nuestras propias creencias, a las experiencias vividas, a la identificación que hemos realizado con alguien torpe, etc.

Para Merleau-Ponty (citado en López Sáenz, 2002), el esquema corporal no es una representación estática de las distintas partes del cuerpo, sino la integración dinámica de las mismas en los proyectos motores del organismo. Es "postura orientada hacia determi- 
nada tarea actual o posible", que unifica los horizontes del movimiento actual y del presente vivido. Estos horizontes también están constituidos por imágenes. El esquema del cuerpo propio es condición de nuestra situación y media entre lo interno y lo externo; es la base del cuerpo fenoménico o vivido, el cual existe activamente y es inseparable del mundo y de las relaciones con los otros. El esquema corporal es un léxico de corporeidad en el quese interrelacionan las distintas intersubjetividades tanto propias como ajenas.

Gallego del Castillo (2009) después de su análisis concluye que esquema corporal es "un lugar de encuentro de conocimientos, pensamientos, percepciones, sensaciones, emociones, sentimientos y de otros aspectos subjetivos o conductuales, que se manifiestan en el movimiento (con su conocimiento y puesta en acción), en el manejo de objetos y en la relación con los demás".

\section{A MODO DE CONCLUSIÓN}

Atendiendo a la conceptualización de cada uno de los términos según las diferentes disciplinas, Thompson, Heingerg, Altabe y Tantleff-Dunn (1998) recogen una serie de definiciones de imagen corporal y términos cercanos concluyendo que el uso de una expresión u otra depende más de la orientación científica de la investigación, del aspecto concreto de la imagen corporal que se va a investigar o incluso de la técnica de evaluación disponible.

Sassano (2003, p. 152), que estudió la evolución de los conceptos de esquema corporal e imagen corporal confrontando a más de 20 autores, dice que: " $a$ pesar de la variada terminología que se emplea para designar este tema, no hay excesivas diferencias entre unas posturas y otras, y las diversas interpretaciones que se dan más bien se complementan unas a otras". Como diría el autor, la confusión proviene de que a veces se usan los mismos términos con significaciones muy diferentes".
Según diferentes autores, no existe hoy en día una definición fiable, estricta y rigurosa de lo que es la imagen corporal. Sin embargo, todos coinciden en señalar que se trata de un constructo multidimensional que abarca desde los pensamientos y creencias del propio cuerpo hasta las autopercepciones, sentimientos y acciones referentes al mismo. La definición más clásica de imagen corporal la describe como "la representación del cuerpo que cada individuo construye en su mente. Desde el punto de vista neurológico, la imagen corporal constituye una representación mental diagramática de la conciencia corporal de cada persona. Una concepción más dinámica define a la imagen corporal en términos de sentimientos y actitudes hacia el propio cuerpo. Algunos autores advierten de la necesidad de distinguir entre representación interna del cuerpo y sentimientos y actitudes hacia él". A estos aspectos debemos añadirle los factores históricos, sociales, culturales, individuales y biológicos que menciona Slade (1994) y que influyen en la construcción o formación de nuestra imagen corporal (Baile Ayensa, 2003; Pastor Gil, 2004; Raich, 2004).

Por lo tanto y para terminar entendemos que son dos las principales conclusiones de nuestra investigación:

1. Cuando hablamos de imagen corporal, hablamos de identidad corporal, hablamos de corporeidad, hablamos de cuerpo. Hablamos de todos los elementos y factores relacionados con el cuerpo y con la imagen que nos formamos personalmente y que reflejamos exteriormente.

2. La imagen corporal debe ser definida como un constructo multidimensional en el que intervienen desde aspectos perceptivos, cognitivos, emocionales y conductuales, así como factores históricos, culturales e individuales, que influyen en determinadas alteraciones psicológicas como la distorsión, la insatisfacción, la presión corporal e incluso la ejecución de determinadas actuaciones perjudiciales para la salud.

\section{BIBLIOGRAFÍA}

Aréchaga, A. J. (2010). El cuerpo y las desigualdades sociales: el espiral de la reproducción social. Revista Latinoamericana de Estudios sobre Cuerpos, Emociones y Sociedad, 2 (2), pp. 16-26. Disponible en http://www.relaces.com.ar/index.php/ relaces/article/view/52/39

Arostegi, B. (2013). Características físicas y psicológicas de jóvenes futbolistas de alto rendimiento. [Tesis doctoral inédita]. Leioa: Universidad del País Vasco / Euskal Herriko Unibersitatea.

Astacio, M. (2001). ¿Qué es un cuerpo? A Parte Rei. Revista de Filosofía, 14, pp. 1-4. Disponible en http://serbal.pntic. mec.es/ cmunoz11/cuerpoasta.pdf

Axpe, I. (2012). Diseño y evaluación de un programa educativo para la mejora del autoconcepto físico. [Tesis doctoral inédita]. Leioa: Universidad del País Vasco / Euskal Herriko Unibersitatea.

Baile Ayensa, J. I. (2003). ¿Qué es la imagen corporal? Cuadernos del Marqués de San Adrián: Revista de Humanidades, 2, pp. 53-70. Disponible en http://www.unedtudela.es/archivos_publicos/qweb_paginas/4469/revista2articulo3.pdf 
Barbero González, J. I. (1998). La cultura del consumo, el cuerpo y la Educación Física. Educación Física y Deporte, 20 (1), pp. 9-30. Disponible en http://aprendeenlinea.udea.edu.co/revistas/index. php/educacionfisicaydeporte/article/ view/3387/3150

Barbero González, J. I. (2005). La escolarización del cuerpo: reflexiones en torno a la levedad de los valores del capital "cuerpo» en Educación Física. Revista Iberoamericana de Educación, 39, pp. 25-51. Disponible en http://rieoei.org/ rie39a01.pdf

Barbero González, J. I. (2007). Capital(es) corporal(es) que configuran las corrientes y/o contenidos de la Educación Física escolar. Ágora para la EF y el Deporte, 4-5, pp. 21-38. Disponible en https://www5.uva.es/agora/revista/4/ agora4-5_barbero_2.pdf

Belden, A. K. (2010). As seen on TV: The relationship between body image and cultivation. [Tesis doctoral inédita]. Oklahoma State Univesity. Disponible en http://citeseerx.ist.psu.edu/viewdoc/download?doi=10.1.1.427.8342\& $r$ ep=rep $1 \&$ type $=$ pdf

Benjumea Pérez, M. M. (2010). La motricidad como dimensión humana -un abordaje transdisciplinar-. EspañaColombia: Instituto Internacional de Saber. Disponible en http://viref.udea. edu.co/contenido/publicaciones/motricidad_dimension_humana.pdf

Bruch, H. (1962). Perceptual and conceptual disturbances in anorexia nervosa. Psychosomatic Medicine, 24 (2), pp. 187194. https://doi.org/10.1097/00006842196203000-00009

Cardenal, V. y Fierro, A. (2003). Componentes y correlatos del autoconcepto en la escala de Piers-Harris. Estudios de Psicología, 24 (1), pp. 101-112. https://doi. org/10.1174/021093903321329094

Carrión, J. (2010, 8 de febrero). El cuerpo, querido Watson. El País.

Cash, T. F. (2004). Body image: past, present and future. Body Image, 1, pp. 1-5. https://doi.org/10.1016/S17401445(03)00011-1

Colás Bravo, P. (2007). La construcción de la identidad de género: enfoques teóricos para fundamentar la investigación e intervención educativa. Revista de Investigación Educativa, 25 (1), pp. 151-166. Disponible en http://revistas.um.es/rie/ article/viewFile/96661/92871
Contreras Espinosa, R. S. y González Romo, Z. F. (2010). Los trastornos del comportamiento alimentario y las Ciencias Sociales, una mirada a las revistas científicas. Revista de Investigación Icono14, 8, pp. 9-24. Disponible en www. icono14.net

Cooper-Molinero, A. K. (2011). A study of body image perceptions related to appearance, fitness and health orientation among black women. [Tesis doctoral inédita]. Capella University. Disponible en http://gradworks.umi. com/34/39/3439416.html

Córdoba, M. (2010). La cirugía estética como práctica sociocultural distintiva: un lacerante encuentro entre corporeidad e imaginario social. Revista Latinoamericana de Estudios sobre Cuerpos, Emociones y Sociedad, 2 (2), pp. 16-26. Disponible en http://www. relaces.com.ar/

Costa, M. (2006). La propuesta de MerleauPonty y el dualismo mente/cuerpo en la tradición filosófica. A Parte Rei. Revista de Filosofía, 47, pp. 1-7. Disponible en http://serbal.pntic.mec.es/ cmunoz11/ malena47.pdf

Cruz, S. y Maganto, C. (2002). Índice de masa corporal, preocupación por la delgadez y satisfacción corporal en adolescentes. Revista de Psicología General y Aplicada, 55 (3), pp. 455-473. Disponible en http://www.sc.ehu.es/ptwmamac/articulos/24a.pdf

De la Cruz Vives, M. Á. (2002). El problema cuerpo-mente: distintos planteamientos. Boletín REDcientifica, 49. Disponible en http://www.redcientifica.com/

Dewar, A. (1994). El cuerpo marcado por el género en la Educación Física: Una perspectiva feminista crítica. En Barbero, J. I. (coord.) Investigación alternativa en Educación física: II Encuentro Unisport sobre Sociología del Deporte. Málaga: Junta de Andalucía, pp. 111-125.

Dolto, F. (1994). La imagen inconsciente del cuerpo. Madrid: Paidós.

Eisenberg, R. (coord.) (2003). Corporeidad, movimiento y educación física. México: Consejo Mexicano de Investigación Educativa.

Esnaola, I. (2005). Elaboración y validación del cuestionario AFI (Autokontzeptu Fisikoaren Itaunketa) de autoconcepto físico. [Tesis doctoral inédita]. Leioa: Universidad del País Vasco / Euskal Herriko Unibersitatea.
Esnaola, I. (2008). El autoconcepto físico durante el ciclo vital. Anales de Psicología, 24 (1), pp. 1-8. Disponible en http://www. um.es/analesps/v24/v24_1/01-24_1.pdf

Esnaola, I., Goñi, A. y Madariaga, J. M. (2008). El autoconcepto: perspectivas de investigación. Revista de Psicodidáctica, 13 (1), pp. 69-96. Disponible en http://www.redalyc.org/ pdf/175/17513105.pdf

Esnaola, I. y Revuelta, L. (2009). Relaciones entre la actividad física, autoconcepto físico, expectativas, valor percibido y dificultad percibida. Acción Psicológica, 6 (2), pp. 31-43. http://dx.doi. org/10.5944/ap.6.2.219

Esteve Rodrigo, J. V., Musitu Ochoa, G. y Lila Murillo, M. (2005). Autoconcepto físico y motivación deportiva en chicos y chicas adolescentes. La influencia de la familia y de los iguales. Escritos de Psicología, 7, pp. 82-90. Disponible en http://www.escritosdepsicologia.es/ descargas/revistas/num7/escritospsicologia7_investigacion.pdf

Faria, L. (2005). Desenvolvimento do auto-conceito físico nas crianças e nos adolescentes. Análise Psicológica, 4 (23), pp. 361-371. Disponible en https://repositorio-aberto.up.pt/bitstream/10216/5484/2/82434.pdf

Ferguson, C. (2011). The relationship between American media exposure and Trinidadian female adolescents' body image satisfaction. [Tesis doctoral inédita]. University of South Florida. Disponible en http://scholarcommons.usf.edu/cgi/viewcontent. cgi $?$ article $=4295 \&$ context $=$ etd

Fernández Zabala, A. (2010). El autoconcepto social: componentes, desarrollo y medida. [Tesis doctoral inédita]. Leioa: Universidad del País Vasco / Euskal Herriko Unibersitatea.

Firenze, A. (2003). El problema de la percepción y la fenomenología de Merleau-Ponty. NODVS. L'aperiòdic virtual de la Secció Clínica de Barcelona, pp. 1-7. Disponible en http://www. scb-icf.net/nodus/contingut/arxiupdf. php? idarticle $=97 \&$ rev $=20$

Galiano, D., Porta, J. y Tejedo, A. (2003). Forma, cuerpo y función. Cádiz: Consejería de Turismo y Deporte. Junta de Andalucía.

Gallego del Castillo, F. (2009). Esquema corporal e imagen corporal. Revista Española de Educación Física y Deportes, 
12, pp. 45-63. Disponible en http:// www.reefd.es/index.php/reefd/article/ view/310/300

García Alonso, A. (2011). Vigorexia. La prisión corporal. Madrid: Pirámide.

González Fernández, O. (2011). La presión sociocultural percibida sobre el autoconcepto físico. Medida y variabilidad. [Tesis doctoral inédita]. Leioa: Universidad del País Vasco / Euskal Herriko Unibersitatea.

Goñi, E. (2009). El autoconcepto personal: estructura y variabilidad. [Tesis doctoral inédita]. Leioa: Universidad del País Vasco / Euskal Herriko Unibersitatea.

Goñi, A., Ruiz de Azúa García, S. y Rodríguez Fernández, A. (2005). La importancia conferida a los diversos aspectos del yo físico: Un instrumento para su medida. Anales de Psicología, 21 (1), pp. 92-101. Disponible en http://revistas.um.es/ analesps/article/viewFile/27201/26381

Grasso, A. (2001). El aprendizaje no resuelto de la Educación Física. La corporeidad. Buenos Aires: Novedades Educativas.

Grasso, A. (2006). Construyendo el concepto de corporeidad. En Brito, L. F., Palacios, L. E., Martínez Jasso, C. y Tinoco Sánchez, R. (comps.). Primer Taller de Actualización sobre los Programas de Estudio 2006. Educación Física I. Antología. México: Secretaría de Educación Pública, pp. 25-37.

Grasso, A. (2008). La palabra corporeidad en el diccionario de Educación Física. Revista Digital Portal deportivo, 1 (4), pp. 1-7.

Heredia, N. y Espejo, G. (2009). Historia de la belleza. Acta de Otorrinolaringología \& Cirugía de Cabeza y Cuello, 37 (1), pp. 31-46.

Infante, G. (2009). Actividad física y autoconcepto físico en la edad adulta. [Tesis doctoral inédita]. Leioa: Universidad del País Vasco / Euskal Herriko Unibersitatea.

Joshi, A. (2011). Participation in extracurricular activities as a protective factor against negative body image. [Tesis doctoral inédita]. University of Hartford. Disponible en http://gradworks. umi.com/34/38/3438013.html

Kogan, L. (2011). Jóvenes y viejos: ¿el cuerpo como locus de identidad? Revista Latinoamericana de Estudios sobre Cuerpos, Emociones y Sociedad, 5, pp. 15-24. Disponible en http://www.relaces.com.ar/index.php/relaces/article/ viewFile/120/72
Le Boulch, J. (2001). El cuerpo en la escuela del siglo XXI. Barcelona: Inde.

León París, C. (2000). Influencia del sexo en la práctica deportiva. Biología de la mujer deportista. Arbor, 165 (650), pp. 249-263. Disponible en http://arbor.revistas.csic.es/index.php/arbor/article/ viewFile/968/975

López Sáenz, M. C. (2002). La existencia como corporeidad y carnalidad en la filosofía de M. Merleau-Ponty. En López Sáenz, M. C. y Rivera de Rosales Chacón, J. C. (coords.) El cuerpo. Perspectivas filosóficas. Madrid: UNED, pp. 308-341.

Marín, M. A. (2002). La construcción de la identidad en la época de la mundialización y los nacionalismos. En Bartolomé, M. (coord.). La construcción de la identidad en contextos multiculturales. Madrid: CIDE.

Martín Serrano, M. (2002). Las transiciones juveniles y las contradicciones de la socialización. Ponencia presentada en la Conferencia Europea sobre Jóvenes y Políticas de Transición en Europa. Madrid, Junio 2002.

Pastor Pradillo, J. L. (2002). Fundamentación conceptual para una intervención psicomotriz en Educación Física. Barcelona: Inde.

Pastor Pradillo, J. L. (2008). La identidad como elemento común del espacio profesional y el ámbito académico. Revista Fuentes, 8, pp. 36-52. Disponible en https://ojs.publius.us.es/ojs/index.php/ fuentes/article/view/2455

Pastor Gil, R. (2004). Cuerpo y género. Representación e imagen corporal. En Barberá Heredia, E., Martínez Benlloch, I. y Bonilla Campos, A. (coords.). Psicología y género. Madrid: Pearson Educación, pp. 218-239.

Pazos, J. M. y Rey, A. (2000). La corporeidad. En Trigo, E. (coord.). Fundamentos de la motricidad. Madrid: Gymnos, pp. 75-87.

Pérez Riobello, A. (2008). Merleau-Ponty: percepción, corporalidad y mundo. Eikasia. Revista de Filosofía, 4 (20), pp. 197-220. Disponible en https:// www.researchgate.net/publication/28230156_Merleau-Ponty_percepcion_corporalidad_y_mundo

Petty, K. (2011). Implicit theories, dissatisfaction and risk behavior: A new theoretical approach to body. [Tesis doctoral inédita]. University of Houston.
Disponible en http://search.proquest. com/docview/608113749

Pruzinsky, T. y Cash, T. F. (2002). Understanding body images: Historical and contemporary perspectives. En Cash,T. F. y Pruzinsky, T. (eds.). Body image: $A$ handbook of theory, research and clinical practice. New York: Guilford Press, pp. 3-12.

Raich, R. M. (1994). Anorexia y bulimia: trastornos alimentarios. Barcelona: Pirámide.

Raich, R. M. (2004). Una perspectiva desde la psicología de la salud de la imagen corporal. Avances en Psicología Latinoamericana, 22, pp. 15-27. Disponible en http:// www.urosario.edu.co/medicina/apl/

Revuelta, L. (2012). El autoconcepto físico y la motivación físico-deportiva durante la adolescencia. [Tesis doctoral inédita]. Leioa: Universidad del País Vasco / Euskal Herriko Unibersitatea.

Rigobello, A. (2000). El porqué de la filosofía. Madrid: Caparrós Editores.

Rima, B. N. (2011). Women's Body Image and Health: A Feminist Intervention Approach. [Tesis doctoral inédita]. The George Washington University. Disponible en http://pqdtopen.proquest. com/doc/848932422.html?FMT=Al

Rodríguez Fernández, A. (2008). El autoconcepto físico y el bienestar/malestar psicológico. [Tesis doctoral inédita]. Leioa: Universidad del País Vasco / Euskal Herriko Unibersitatea.

Rodríguez Fernández, A. (2009). Autoconcepto físico y bienestar/malestar psicológico en la adolescencia. Revista de Psicodidáctica, 14 (1), pp. 155-158. Disponible en http://www.redalyc.org/ articulo.oa?id=17512723011

Rodríguez, N. y Galak, E. (2009). Sinécdoque de un autor: habitus y cuerpo en Pierre Bourdieu. Entrevista a Alicia Gutiérrez. Cuadernos de H ideas, 3 (3), pp. 1-9. Disponible en http://perio.unlp.edu.ar/ojs/ index.php/cps/article/view/1378/1694

Ruiz de Azúa, S. (2007). El autoconcepto físico: estructura interna, medida y variabilidad. [Tesis doctoral inédita]. Leioa: Universidad del País Vasco / Euskal Herriko Unibersitatea.

Ruiz Calvente, M. (2008). Cuerpo, Realidad y Expresión. A Parte Rei. Revista de Filosofía, 59, pp. 1-13. Disponible en http:// serbal.pntic.mec.es/ cmunoz11/calvente59.pdf 
Sassano, M. (2003). Cuerpo, tiempo y espacio. Principios básicos de la psicomotricidad. Buenos Aires: Stadium.

Schilder, P. (1989). Imagen y apariencia del cuerpo humano. México: Paidos.

Sierra Hernando, C. H. (2007). De la mirada pétrea a la mirada encarnada: MerleauPonty y la invisibilidad del cuerpo. En Arpal Poblador, J. y Mendiola, I. Estudios sobre cuerpo, tecnología y cultura. Vizcaya: Servicio de Publicaciones. Universidad del País Vasco, pp. 147-166.

Slade, P. D. (1973). A Short Anorexic Behaviour Scale. British Journal of Psychiatry, 122 (566), pp. 83-85. https://doi. org/10.1192/bjp.122.1.83

Slade, P. D. (1994). What is body image? Behaviour Research and Thera- py, 32 (5), pp. 497-502. https://doi org/10.1016/0005-7967(94)90136-8

Sossa, A. (2009). Cuerpo y sociología. Reflexiones sobre el cuerpo en la teoría sociológica clásica. Exploración al pensamiento de Marx, Durkheim y Weber. Revista Cultura y Religión, 3 (1), pp. 167-183. Disponible en https://dialnet.unirioja.es/servlet/ articulo?codigo $=2952662$

Thompson, J. K., Heinberg, L. J., Altabe, M. N. y Tantleff-Dunn, S. (1998). Exacting beauty: Theory, assessment and treatment of body image disturbance. Washington D.C.: American Psychological Association.

Trigo, E. (coord.) (2000). Fundamentos de la motricidad. Madrid: Gymnos.
Vázquez, B. (1989). La Educación Física en la educación básica. Madrid: Gymnos.

Vázquez, B. (coord.) (2001). Bases educativas de la actividad física y el deporte. Madrid: Síntesis.

Zubeldia, M. (2015). El autoconcepto musical, motivación y bienestar psicológico del alumnado de Conservatorio. [Tesis doctoral inédita]. Leioa: Universidad del País Vasco / Euskal Herriko Unibersitatea.

Zulaika, L. (1999). Análisis relacionales y eficacia de un programa de intervención para la mejora del autoconcepto físico. [Tesis doctoral inédita]. Leioa: Universidad del País Vasco / Euskal Herriko Unibersitatea. 